\title{
COMMUNICATIONS
}

\section{3-Second FLASH MRI of the Human Heart}

\author{
J. Frahm, K. D. Merboldt, H. Bruhn, M. L. Gyngell, \\ W. HÄNICKE, AND D. CHIEN \\ Max-Planck-Institut für biophysikalische Chemie, Posifach 2841, \\ D-3400 Göttingen, Federal Republic of Germany
}

Received June 26, 1989; revised October 10, 1989

\begin{abstract}
Flow-suppressed FLASH MR images of the human heart have been recorded within a measuring time of $0.3 \mathrm{~s}$ using a 2.0-T whole-body research system (Siemens Magnetom) equipped with a conventional $10 \mathrm{mT} \mathrm{m}^{-1}$ gradient system. Subsecond imaging times have been achieved by reducing the repetition time to $T R=4.8 \mathrm{~ms}$ and by lowering the spatial resolution to $64 \times 128$ measured data points. The flip angle of the slice-selective radiofrequency $(\mathrm{rf})$ pulses was adjusted to $10^{\circ}$. Cardiac chambers, ventricular walls, and valves are well delineated in images from a single cardiac cycle using a field of view of 250 $\mathrm{mm}$ and a slice thickness of $8 \mathrm{~mm}$. No motion artifacts were observed as a consequence of the short echo time of TE $=2.8 \mathrm{~ms}$. Distinction between flowing blood and solid structures has been achieved by spatial presaturation of adjacent slices using two slice-selective $90^{\circ}$ rf pulses preceding the entire imaging sequence. () 1990 Academic Press, Inc.
\end{abstract}

\section{INTRODUCTION}

Functional investigations of the heart represent the most challenging application of magnetic resonance imaging (MRI) as they simultaneously require high spatial resolution and time resolution. While conventional ECG-synchronized spin-echo MRI sequences offer excellent static anatomical details at the expense of considerable measuring times, see, e.g., (1), dynamic information is available from fast scan FLASH MRI sequences $(2,3)$ in the form of multiphase ECG-synchronized cine MR studies, see, e.g., (4). However, both approaches may suffer from the fact that the image data are acquired from different cardiac cycles. One solution to this problem, true "snapshot" echo-planar imaging (EPI), has been developed by Mansfield et al. $(5-7)$ and implemented to a 2.0-T field strength by Pykett and Rzedzian $(8,9)$. Typical imaging times are $64 \mathrm{~ms}$ for a $64 \times 128$ pixel resolution image $(10)$.

In contrast to EPI where spins are excited only once and data are encoded by very fast gradient reversals or oscillations, this communication deals with an adaptation of the FLASH principles to allow subsecond MRI studies of the heart. Recently, a fast $T_{1}$ - or $T_{2}$-weighted FLASH sequence has been demonstrated to provide rapidly adjustable contrasts in phantoms and human extremities at $4.7 \mathrm{~T}(11)$. However, in our opinion the only real justification for a high-speed MRI technique with its unavoidable trade-offs in spatial resolution and signal-to-noise must be its ability to acquire snapshot images of the beating heart. In fact, complications from patients with cardiac arrhythmia and concomitantly difficult ECG patterns can only be avoided if all the data are acquired from a single cardiac cycle, thus limiting the mea- 


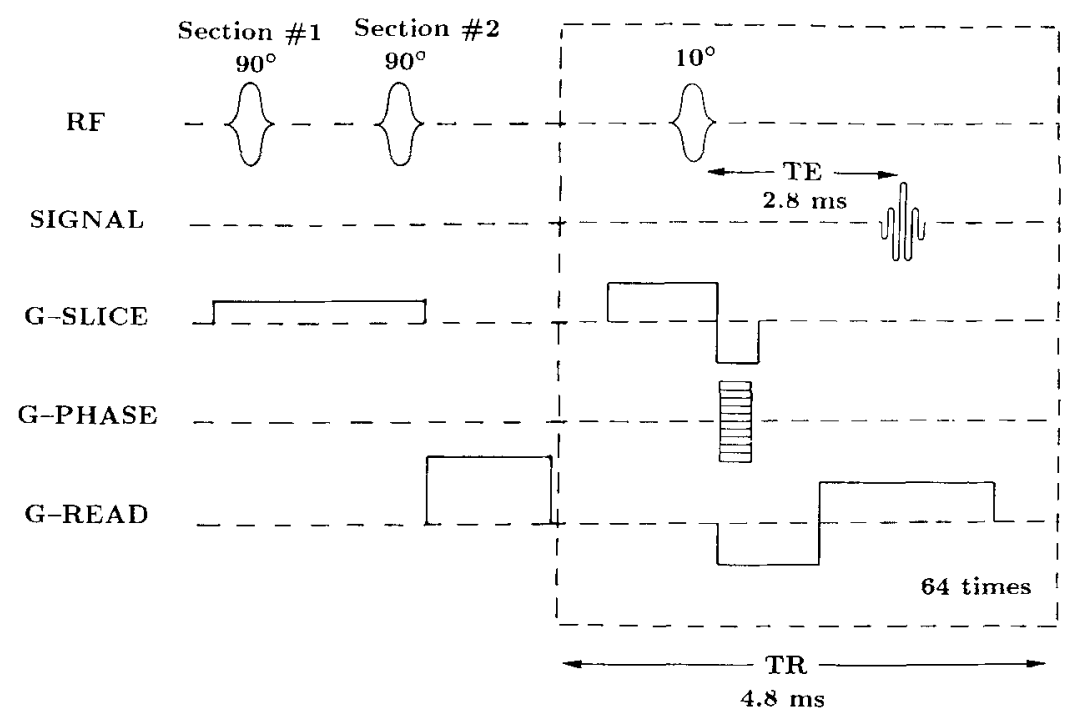

FIG. 1. Schematic diagram of the radiofrequency ( $\mathrm{rf}$ ) and magnetic field gradient sequence used for $0.3-$ s FLASH MRI of the heart. For details see text.

suring time to a fraction of it. In other parts of the body alternative approaches with longer imaging times, but better spatial resolution and better signal-to-noise, should be preferable.

\section{METHODS}

Figure 1 shows a schematic diagram of the flow-suppressed FLASH sequence used for 0.3-s MRI of the human heart. The repetition time and the echo time were reduced to $\mathrm{TR}=4.8 \mathrm{~ms}$ and $\mathrm{TE}=2.8 \mathrm{~ms}$, respectively. Accordingly, a matrix of 64 $\times 128$ measured complex data points leads to a total measuring time of $307 \mathrm{~ms}$. For display purposes the images were interpolated to a $256 \times 256$ image matrix. Typically, the measured field of view (FOV) was $250 \mathrm{~mm}$ with a slice thickness of $8 \mathrm{~mm}$. The radiofrequency (rf) pulses were sinc-shaped with a duration of $0.64 \mathrm{~ms}$ and a flip angle of $10^{\circ}$. Data were acquired over an acquisition period of $1.92 \mathrm{~ms}$ duration. This corresponds to a receiver bandwidth of $66.7 \mathrm{kHz}$ assuming oversampling in the frequency-encoding dimension.

Spatial presaturation of flowing material (12) was accomplished by exciting a $60-$ $\mathrm{mm}$ slice offset by $35 \mathrm{~mm}$ on each side of the imaging plane. The duration of the two sinc-shaped $90^{\circ} \mathrm{rf}$ pulses was $2.56 \mathrm{~ms}$. Residual transverse magnetizations were dephased by means of a spoiler gradient. The duration of the entire preparation period was about $20 \mathrm{~ms}$. It is immediately followed by image acquisition starting from the full longitudinal equilibrium magnetization without dummy scans. Using flip angles of $10^{\circ}$ a sequence with 64 repetition cycles is finished before a steady-state magnetization has been established.

In this study subsecond FLASH MR images were recorded without ECG triggering using the standard circular polarized body coil. It should also be noted that all images 

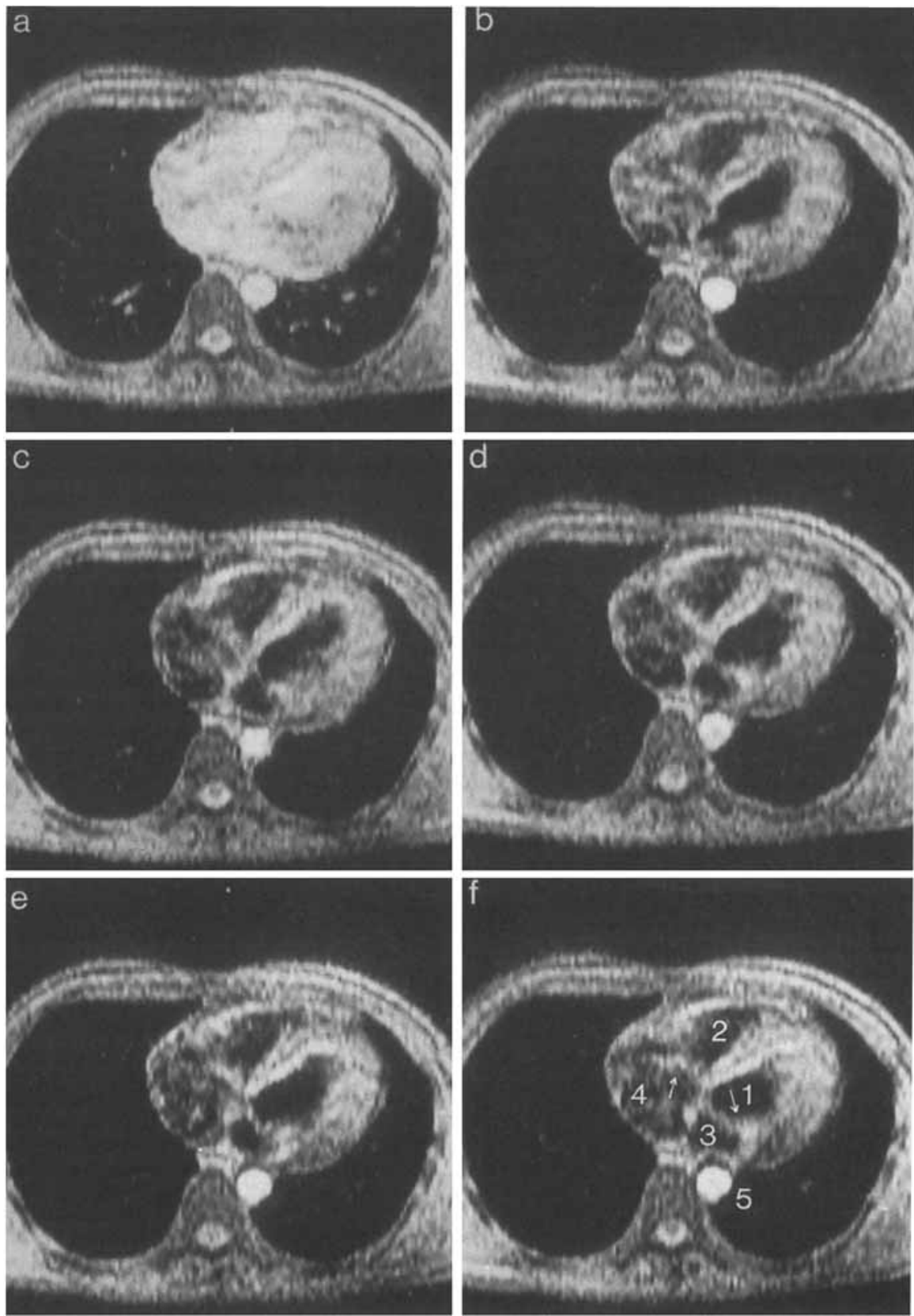

FiG. 2. Eighty-five-megahertz (2.0-T) transverse 0.3-s FLASH MR images of the heart of a normal volunteer obtained using the sequence and the parameters as shown in Fig. 1. The images represent single excitation images and have been recorded using the standard circular polarized body coil. The spatial resolution corresponds to $64 \times 128$ measured data points (interpolated to $256 \times 256$ pixel for display) covering a field of view (FOV) of $250 \mathrm{~mm}$ with a slice thickness of $8 \mathrm{~mm}$. The images were selected out of 
represent single excitation images. Averaging of images from different cardiac cycles has been avoided as this procedure results in a loss of the benefits of true high-speed imaging. Experiments were carried out on a 2.0-T whole-body research system (Siemens Magnetom) equipped with a conventional gradient system yielding a maximum of $10 \mathrm{~m} \mathrm{~T} \mathrm{~m}^{-1}$. Gradient rise and fall times were adjusted to $300 \mu \mathrm{s}$. All investigations were performed on normal volunteers (male, 20-25 years). Informed consent was obtained prior to the investigations.

\section{RESULTS}

The importance of flow suppression for gaining blood/muscle contrast is demonstrated in Fig. 2. Only minor anatomical details can be observed in Fig. 2a where the flip angle of the presaturation pulses has been set to zero. Thus, the contrast between blood and cardiac soft tissues is severely compromised by complex reflow phenomena. A potential clinical value of flow-containing images may be derived from cine representations of images recorded as a function of time during the cardiac cycle. The flow-suppressed images in Figs. 2b-2f are from the same slice as Fig. 2a. In the absence of ECG synchronization they have been selected from a series of nontriggered images from different heartbeats. The images refer to a transverse cross section of the heart at the level of the right and left ventricles, the right and left atrium, the mitral and tricuspid valves, and the descending aorta as indicated in Fig. 2f. Retrospective sorting according to the cardiac cycle resulted in a series of images depicting the opening (Fig. 2b) and closing (Figs. 2e, 2f) of the mitral valve.

Figure 3 shows an equivalent set of 0.3 -s FLASH MR images from a different volunteer. The images refer to a transverse section below the level of Fig. 2 and above the right diaphragm depicting the inferior vena cava. They represent six phases from the cardiac cycle including late diastole (Fig. 3a), early (Fig. 3b) and mid systole (Fig. 3c) exhibiting wall thickening, and subsequent diastolic relaxation (Figs. 3d-3f). Flow signals in the aorta indicate velocities of greater than $0.4 \mathrm{~m} \mathrm{~s}^{-1}$ corresponding to $60-\mathrm{mm}$ saturation and $150-\mathrm{ms}$ reflow. Figure 4 shows a series of diastolic (left) and systolic (right) images at different transverse sections through the heart. The top images (Figs. 4a, 4b) are near the base of the heart showing the pulmonary outflow tract and the ascending aorta. Subsequent images have been recorded $10 \mathrm{~mm}$ (Figs. 4c, 4d), $15 \mathrm{~mm}$ (Figs. 4e, 4f), and $30 \mathrm{~mm}$ (Figs. 4g, 4h) caudally to this plane. The coronary sinus is delineated in Fig. $4 \mathrm{~h}$.

\section{DISCUSSION}

An important result of subsecond FLASH MRI is the absence of motion artifacts. Despite the relatively long measuring time of $0.3 \mathrm{~s}$, without breath holding, and even in systole, the development of phase errors due to motion in the presence of the imaging gradients remains negligible. This is due to the very short echo time of the

a series of images acquired from different heartbeats without ECG triggering. Without flow suppression (a), flow-suppressed images (b)-(f) at different times during the cardiac cycle representing opening (b) and closing (e, $f$ ) of the mitral valve. Numbers: 1 , left ventricle; 2 , right ventricle; 3 , left atrium; 4 , right atrium; 5 , descending aorta. Arrows denote the mitral and tricuspid valves, respectively. 
sequence. View-to-view motions of the heart during the scan may cause image blurring but do not lead to ghosting artifacts. The delineation of cardiac structures in $0.3-$ $s$ images also benefits from the fact that only a minor part of the 64 phase-encoding steps is responsible for the gross image appearance. This feature has been described in detail by Riederer et al. (13) and used for correcting images of moving objects. Image changes during the cardiac cycle are predominantly due to data from the central phase-encoding gradients acquiring the low spatial frequencies. Consequently, the images refer to the heart as seen within a central $50-\mathrm{ms}$ window of the $300-\mathrm{ms}$ imaging sequence. This allows all phases of the cardiac cycle to be covered by images from different heartbeats. Obviously, the demonstration of an effective time resolution better than the total imaging time is further facilitated by means of ECG triggering. Such studies are the subject of a subsequent publication (14).

In this preliminary application the degree of flow suppression was partially compromised by reflow phenomena during the 150 -ms interval between presaturation and the center of the image acquisition. In future studies the efficacy of the saturation pulses may be considerably improved by reordering the phase-encoding steps in such a way as to start from zero and to proceed with alternating positive and negative values. Further applications of flow suppression during the preparation period may deal with single-side or in-plane saturation to determine flow patterns in the heart.

Although subsecond FLASH MRI in its present form is still a factor of 5 slower than EPI, it requires only conventional hardware and standard Fourier imaging algorithms. Even shorter TR/TE values and imaging times of the order of 100-200 ms will become accessible using gradient systems that offer both higher gradient strengths and shorter switching times. In addition, half Fourier techniques (15) may further cut the measuring times or improve the spatial resolution by a factor of 2 , respectively.

Since 0.3-s FLASH images represent data from a single cardiac cycle the present approach gains the same advantages as previously claimed for EPI. In addition, the FLASH sequence offers a variety of other benefits. For example, from a practical point of view, zooming the FOV is much simpler than with EPI where restricting conditions must be met for the two spatially encoding gradients. Similar arguments apply for the recording of oblique orientations as required for most cardiac MRI studies. A further advantage over EPI stems from the fact that chemical-shift artifacts are avoided in the presence of the strong gradients and the correspondingly large receiver bandwidth used here. Finally, it is noteworthy that the present technique is not sensitive to susceptibility artifacts. Such degrading effects may be observed with EPI in particular when an increase of the spatial resolution (data matrix) leads to an increase of the effective gradient echo times.

\section{CONCLUSIONS}

Subsecond FLASH MRI has been demonstrated to yield clear images of the human heart from data collected within a single cardiac cycle. Functional studies of the myocardium are considerably improved by combining the technique with spatial presaturation to eliminate blood flow from the images. Images at different times during the cardiac cycle show myocardial wall thickening and valve movements without motion artifacts. The sequence may record images without ECG synchronization and, there- 

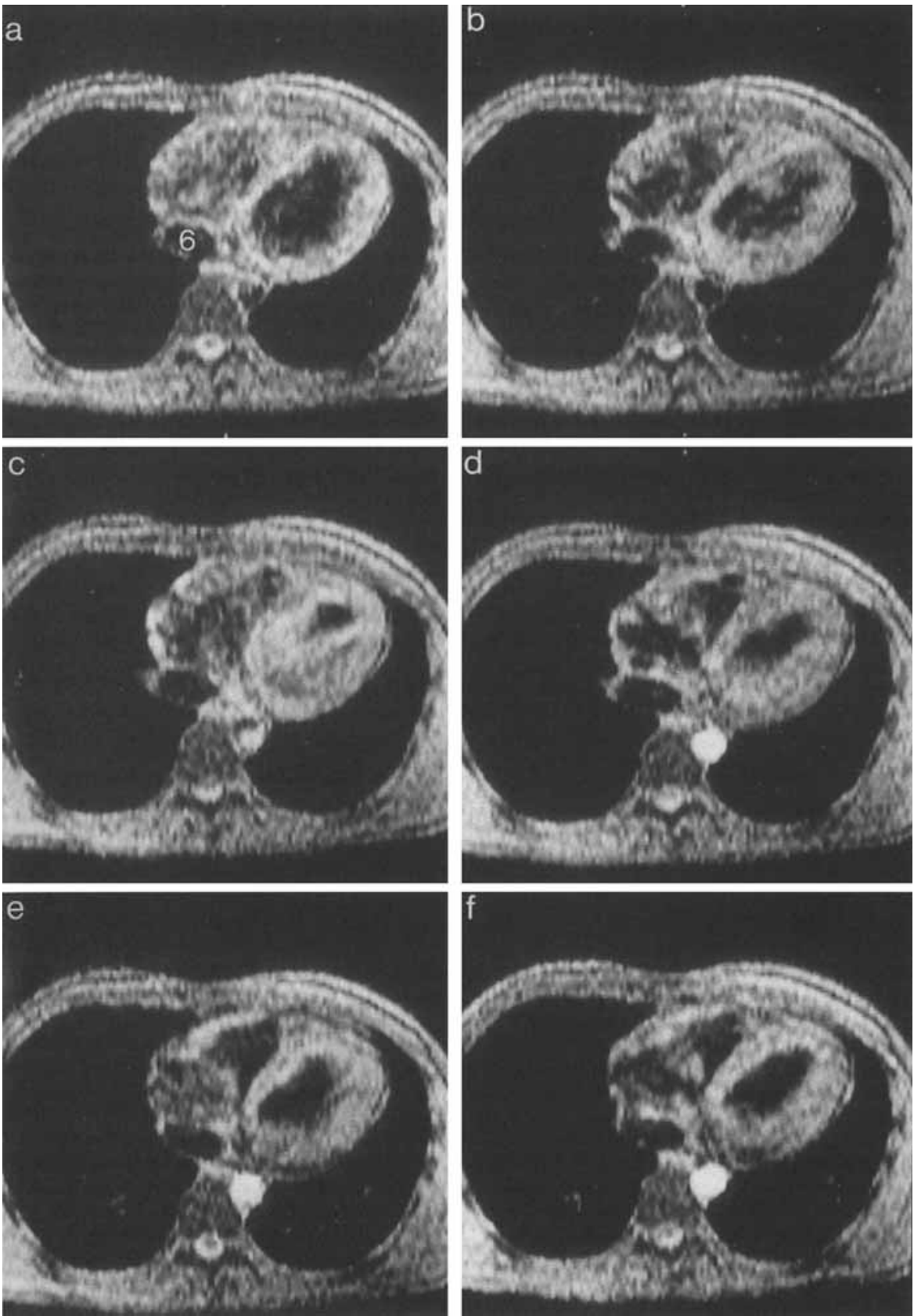

FIG. 3. Eighty-five-megahertz (2.0-T) transverse 0.3-s FLASH MR images of the heart of a normal volunteer. The imaging sequence and all other parameters are as given in Figs. 1 and 2 . The images refer to the same section and represent different events during the cardiac cycle including late diastole (a), early systole (b), mid systole (c), and subsequent diastolic phases (d-f). Number: 6 , inferior vena cava. 

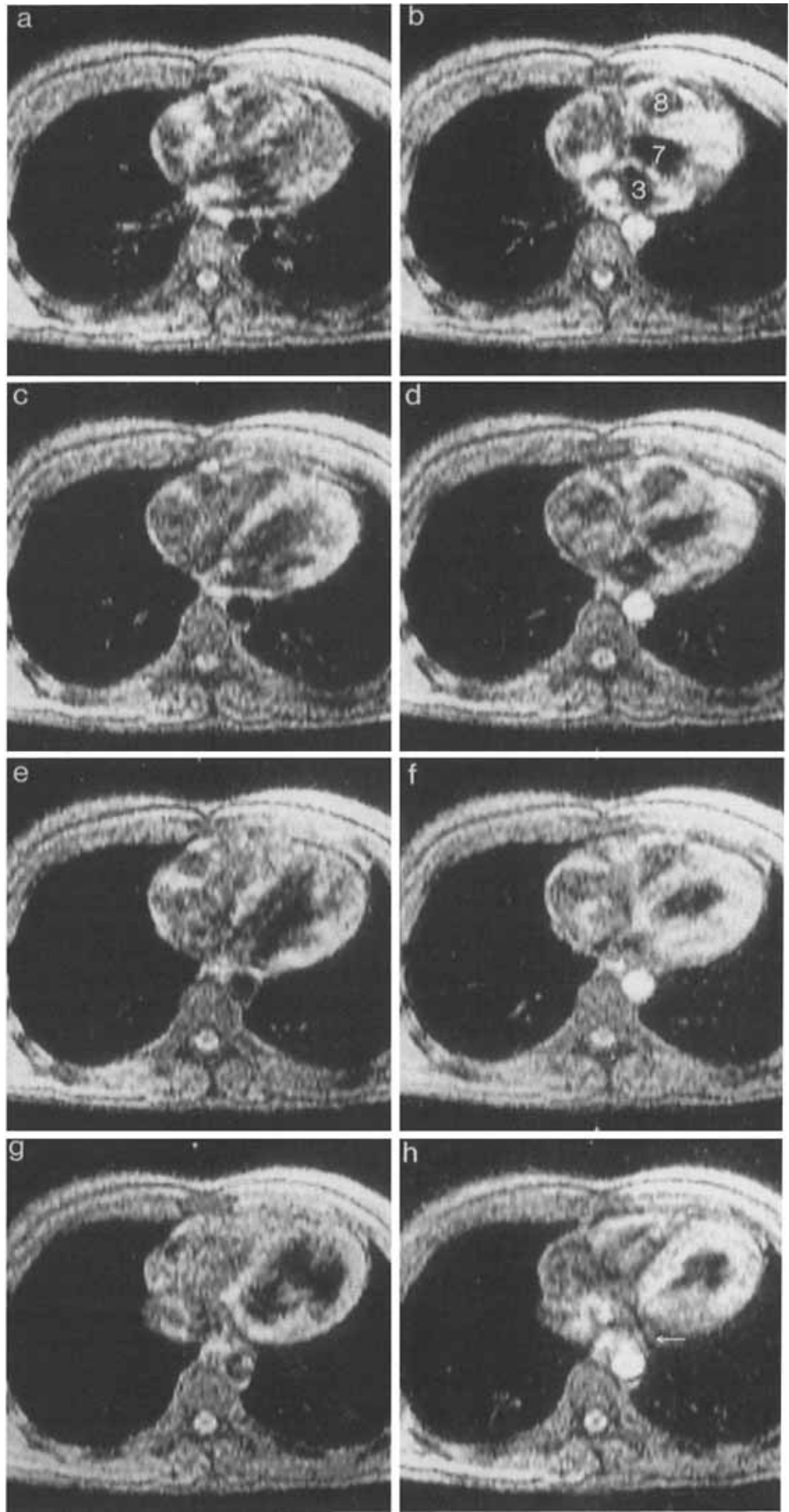

FIG. 4. Eighty-five-megahertz (2.0-T) transverse 0.3-s FLASH MR images of the heart of a normal volunteer. The imaging sequence and all other parameters are as given in Figs. 1 and 2 . The images refer to diastolic (left) and systolic ( right) events in different sections of the heart from top ( $a, b)$ to bottom ( $g$, h). Numbers: 7 , ascending aorta; 8 , pulmonary outflow tract. The arrow denotes the coronary sinus. 
fore, allows the investigation of patients with severe arrhythmia precluding the derivation of a useful trigger signal. Of course, triggered images will simplify a detailed description of the cardiac cycle as well as allow the observation of intercycle variations in patients.

It is important to note that only conventional hardware has been used for $0.3-\mathrm{s}$ FLASH MRI. Improved magnetic field gradient systems are expected to further reduce imaging times. Improvements in image quality are expected by changing the body coil to a more suitable rf coil matching the requirements for cardiac MRI. Whether the method may become competitive to EPI must be evaluated in future studies.

\section{ACKNOWLEDGMENTS}

Financial support by the Bundesminister für Forschung und Technologie (BMFT) of the Federal Republic of Germany (Grant 01 VF 8606/6) is gratefully acknowledged. One of us (D.C.) has been supported by the Fulbright Commission.

\section{REFERENCES}

1. C. B. Higgins and W. AuffermanN, in "Magnetic Resonance Imaging of the Body" (C. B. Higgins and H. Hricak, Eds.), p. 49, Raven Press, New York, 1987; C. B. HigGins, in "Magnetic Resonance Imaging of the Body" (C. B. Higgins and H. Hricak, Eds.), p. 239, Raven Press, New York, 1987.

2. A. HaAse, J. Frahm, D. Matthaei, W. Hänicke, and K. D. Merboldt, J. Magn. Reson. 67, 258 (1986).

3. J. Frahm, A. HaASE, AND D. Matthaei, Magn. Reson. Med. 3, 321 (1986).

4. C. B. Higgins, U. P. SeChtem, AND P. Pflugfelder, in "Magnetic Resonance Imaging of the Body" (C. B. Higgins and H. Hricak, Eds.), p. 295, Raven Press, New York, 1987.

5. P. Mansfield, J. Phys. C 10, L55 (1977).

6. P. Mansfield and I. L. Pykett, J. Magn. Reson. 29, 355 (1978).

7. A. M. Howseman, M. K. Stehling, B. Chapman, R. Coxon, R. Turner, R. J. Ordidge, M. G. Cawley, P. Glover, P. Mansfield, AND R. E. Coupland, Brit. J. Radiol. 61, 822 (1988).

8. R. Rzedzian ANd I. L. Pykett, Amer. J. Roentgenol. 149, 245 (1987).

9. I. L. PyKett AND R. RzEdzIAN, Magn. Reson. Med. 5, 563 (1987).

10. M. J. Stehling, A. M. Howseman, R. J. Ordidge, B. Chapman, R. Turner, R. Coxon, P. Glover, P. MANSFIEld, AND R. E. Coupland, Radiology 170, 257 (1989).

11. A. HAASE, Magn. Reson. Med. 13 (1989).

12. J. Frahm, W. Hänicke, K. D. Merboldt, And A. HaAse, Magn. Reson. Med. 4, 372 (1987).

13. S. J. Riederer, T. Tasciyan, F. FARzaneh, J. N. Lee, R. C. Wright, and R. J. Herfkens, Magn. Reson. Med. 8, 1 (1988).

14. D. Chien, K. D. Merboldt, W. Hänicke, H. Bruhn, M. L. Gyngell, AND J. Frahm, submitted for publication.

15. P. Margosian And F. SchMitT, SPIE Med. Image Process. 593, 6 ( 1985). 